The University of San Francisco

USF Scholarship: a digital repository @ Gleeson Library |

Geschke Center

Physics and Astronomy

College of Arts and Sciences

9-19-2005

\title{
Effective Field Theory Program for Conformal Quantum Anomalies
}

Horacio E. Camblong

University of San Francisco, camblong@usfca.edu

Follow this and additional works at: http://repository.usfca.edu/phys

Part of the Physics Commons

\section{Recommended Citation}

Camblong, Horacio E., "Effective Field Theory Program for Conformal Quantum Anomalies" (2005). Physics and Astronomy. Paper 14. http://repository.usfca.edu/phys/14

This Article is brought to you for free and open access by the College of Arts and Sciences at USF Scholarship: a digital repository @ Gleeson Library | Geschke Center. It has been accepted for inclusion in Physics and Astronomy by an authorized administrator of USF Scholarship: a digital repository @ Gleeson Library | Geschke Center. For more information, please contact repository@usfca.edu. 


\title{
Effective field theory program for conformal quantum anomalies
}

\author{
Horacio E. Camblong, ${ }^{1}$ Luis N. Epele, ${ }^{2}$ Huner Fanchiotti, ${ }^{2}$ Carlos A. García Canal, ${ }^{2}$ and Carlos R. Ordóñez ${ }^{3}$ \\ ${ }^{1}$ Department of Physics, University of San Francisco, San Francisco, California 94117-1080, USA \\ ${ }^{2}$ Laboratorio de Física Teórica, Departamento de Física, Facultad de Ciencias Exactas, Universidad Nacional de La Plata, \\ C.C. 67-1900 La Plata, Argentina \\ ${ }^{3}$ Department of Physics, University of Houston, Houston, Texas 77204-5506, USA
}

(Received 20 April 2005; published 19 September 2005)

\begin{abstract}
The emergence of conformal states is established for any problem involving a domain of scales where the long-range $\mathrm{SO}(2,1)$ conformally invariant interaction is applicable. Whenever a clear-cut separation of ultraviolet and infrared cutoffs is in place, this renormalization mechanism is capable of producing binding in the strong-coupling regime. A realization of this phenomenon, in the form of dipole-bound anions, is discussed.
\end{abstract}

DOI: 10.1103/PhysRevA.72.032107

\section{INTRODUCTION}

The renormalization program [1] provides an insightful framework for the description of physical scales within a given problem. This assumes that the characteristic dimensional scales are sufficiently separated, as required by effective field theory $[1,2]$. Moreover, symmetry considerations usually furnish further analytical control over what contributing factors might be relevant for the hierarchy of scales. In addition to the well-known examples in high-energy physics and condensed matter physics, an effective renormalization of a system in molecular physics was introduced in Ref. [3], where a symmetry-centered approach was developed for the formation of dipole-bound anions by electron capture. In the relevant domain of scales, the dominant physics-governed by an inverse square potential [4] — takes a scale-invariant form known as conformal quantum mechanics.

The central purpose of this paper is to develop an effective field theory program for the quantum anomaly of Ref. [3]. Specifically, we address the role played by additional degrees of freedom-for example, the rotational ones in the molecular case. In this manner, we extensively use recent work on the renormalization and anomalous symmetry breaking of conformal quantum mechanics [5]. As a consequence, we will establish the following results.

(i) The conformal analysis is robust and fairly insensitive to the ultraviolet and infrared physics.

(ii) The effective field approach-centered on renormalization techniques-sheds light, e.g., on the properties of dipole-bound anions; this is in sharp contrast with the statements of Ref. [6].

(iii) The origin of a critical dipole moment for binding can be directly traced to the conformal interaction.

In short, the predictions of the conformal framework of Ref. [3] are not significantly altered by the inclusion of additional degrees of freedom. Moreover, a similar analysis can be applied to other problems for which the conformal quantum anomaly is relevant, for example, for the Efimov effect [7].

\section{CONFORMAL QUANTUM MECHANICS AND DIPOLE-BOUND STATES}

In this section, we start by summarizing the results of Ref. [3] for dipole-bound states in the language of effective field theory [5]. As we will see in the next section, the effective field approach also provides the natural connection between this work and the standard results of rotationally adiabatic theory [8-10].

\section{A. Conformal physics of dipole-bound states}

The dominant part of the electron-molecule interaction can be described with a point dipole - the electron does not significantly probe radial scales smaller than the size $a$ of the molecule. Then, in three spatial dimensions, the associated anisotropic Hamiltonian reads

$$
H=\frac{p^{2}}{2 m_{e}}-\frac{g}{r^{2}} \cos \theta,
$$

in which the coupling $g$ can be recast into a dimensionless form $\lambda=2 m_{e} g / \hbar^{2}$. Under time reparametrizations, this system displays an $\mathrm{SO}(2,1)$ conformal symmetry, whose breaking at the quantum-mechanical level can be interpreted as a quantum anomaly. As a first step, introducing separation of variables: $\Psi(r, \Omega)=u(r) \Xi(\Omega) / r$ in spherical coordinates. This leads to a scale-invariant radial equation

$$
\frac{d^{2} u(r)}{d r^{2}}+\left[k^{2}+\frac{\gamma(\lambda)}{r^{2}}\right] u(r)=0
$$

coupled to a scale-independent angular operator equation

$$
\hat{A}(\lambda) \Xi(\Omega)=\gamma(\lambda) \Xi(\Omega),
$$

where the eigenvalue $\gamma \equiv \gamma(\lambda)$ plays the role of a separation constant and

$$
\hat{A}(\lambda)=-\mathbf{l}^{2}+\lambda \cos \theta,
$$

with $\mathbf{l}$ being the relative orbital angular momentum of the electron about the molecule. The problem defined by the equations above is completely characterized by the solutions of conformal quantum mechanics.

\section{B. Radial conformal quantum mechanics}

Conformal quantum mechanics applies to the description of the radial problem. All the properties and conclusions dis- 
cussed herein rely on the existence of a domain of scales in which the dominant physics is scale invariant. A symmetrycentered analysis in the relevant conformally invariant domain shows that the theory retains the $\mathrm{SO}(2,1)$ symmetry at the quantum level when $\gamma<1 / 4$, with $\gamma=1 / 4$ being a critical point of the conformal framework. The existence of a conformal critical point

$$
\gamma^{(*)} \equiv \gamma\left(\lambda^{(*)}\right)=1 / 4
$$

is the crucial ingredient that explains the experimental fact that electron binding by molecular anions only occurs for dipole moments greater than a critical value $p^{(*)}$ [3].

Conformal quantum mechanics is singular for $\gamma \geqslant 1 / 4$, but can be rescued by the use of renormalization, which yields conformal bound states with energies $E_{n}=E_{0} \exp ($ $-2 \pi n / \Theta)$, where $n$ is a positive integer, $E_{0}$ is the arbitrary ground-state energy, and the conformal parameter $\Theta$ is derived from the coupling according to the rule [5]

$$
\Theta=\sqrt{\gamma-\frac{1}{4}} .
$$

The specific value of the characteristic scale $E_{0}$ defined by the conformal tower of states is sensitive to the ultraviolet physics and cannot be predicted by a renormalization approach alone. However, the scale $E_{0}$ is not relevant in the determination of the relative values of bound-state energies, as exhibited by the geometric scaling

$$
\frac{E_{n^{\prime}}}{E_{n}}=\exp \left[-\frac{2 \pi\left(n^{\prime}-n\right)}{\Theta}\right],
$$

which is a remnant of the original scale invariance. In particular, the geometric ratio $e^{-2 \pi / \Theta}$ of adjacent energy levels has a universal form that is independent of the cutoff and impervious to the ultraviolet physics. Finally, the conformal states are characterized by normalized radial wave functions of the form

$$
u(r)=\kappa \sqrt{\frac{2 \sinh (\pi \Theta)}{\pi \Theta}} \sqrt{r} K_{i \Theta}(\kappa r),
$$

where $K_{i \Theta}(z)$ is the Macdonald function of imaginary index [11]. This is the function whose properties guarantee the universal geometric scaling (7). In addition, the same function leads to an estimate of the characteristic radial size of the electron probability distribution, given by $\kappa^{-1}$, with relative ratios $\kappa_{n} / \kappa_{n^{\prime}}=e^{\pi\left(n^{\prime}-n\right) / \Theta}$ exhibiting a similar kind of universal geometric scaling.

In short, the generic properties of conformal quantum mechanics determine the nature of the bound states of molecular anions and are parametrized by the possible values of the conformal parameter $\Theta$. In turn, $\Theta$ is described, from Eq. (6), in terms of the effective coupling $\gamma=\Theta^{2}+1 / 4$, which is completely determined by the angular dependence of the interaction, through the eigenvalue equation (3). This is the problem to which we now turn.

\section{Angular eigenvalue equation}

The angular problem for an anisotropic conformal interaction is given by Eq. (3), whose secular-determinant form $D(\gamma, \lambda) \equiv \operatorname{det} M(\gamma, \lambda)=0$ involves the infinite matrix $M(\gamma, \lambda)=-A(\lambda)+\gamma \rrbracket$, with 1 being the identity matrix. In particular, in the angular momentum basis $|l, m\rangle$, the matrix elements $\left\langle\operatorname{lm}|M(\gamma, \lambda)| l^{\prime} m^{\prime}\right\rangle=\delta_{m m^{\prime}} M_{l l^{\prime}}(\gamma, \lambda ; m)$ are diagonal with respect to $m$, with tridiagonal blocks

$$
M_{l l^{\prime}}(\gamma, \lambda ; m)=[l(l+1)+\gamma] \delta_{l l^{\prime}}-\lambda\left[N_{l}(m) \delta_{l, l^{\prime}-1}+\left(l \leftrightarrow l^{\prime}\right)\right],
$$

where $N_{l}(m)=\sqrt{\left[(l+1)^{2}-m^{2}\right] /[(2 l+1)(2 l+3)]}$. As a result, the secular determinant takes the factorized form $D(\gamma, \lambda)$ $=\Pi_{m} D_{m}(\gamma, \lambda)$ and the eigenvalues are given by the roots of the reduced determinants $D_{m}(\gamma, \lambda) \equiv \operatorname{det}\left[M_{l l^{\prime}}(\gamma, \lambda ; m)\right]=0$, for all integer values of $m$. At this purely conformal level, for every $m$, the roots $\gamma_{h, m}$ can be arranged in a decreasing sequence: $\gamma_{0, m} \geqslant \gamma_{1, m} \geqslant \gamma_{2, m} \geqslant \cdots$, with $h=0,1, \ldots$, and compared against the condition for conformal criticality: $\gamma$ $=\gamma^{(*)}=1 / 4$. Equation (9) implies the following trends: $\gamma$ is a monotonic function with respect to both $\lambda$ and $m$, increasing with $\lambda$ and decreasing with $m$. In particular, for any finite dipole moment $p$ (i.e., finite $\lambda$ ), there exist only a finite number of supercritical values of $\gamma$; in turn, for each $\gamma$, there is an infinite tower of conformal states-possibly limited by the onset of nonconformal physics for long-distance scales. Hence the conformal bound states are completely characterized by the set of quantum numbers $(n, h, m)$, in which the subset $(h, m)$ determines $\gamma_{h, m}$, while $n$ labels the ordering of the conformal tower or geometric scaling. The existence of these states in the "supercritical regime" yields anomalous breaking of the $\mathrm{SO}(2,1)$ commutator algebra [5].

An important related question is: for the largest root $\gamma_{0,0}$, what is the value $\lambda^{(*)}$ that generates a conformal critical point? By setting $\gamma_{0,0}=\gamma^{(*)}=1 / 4$, the "principal conformal critical coupling" becomes $\lambda_{\text {conf }}^{(*)} \approx 1.279$ whence the required critical dipole moment is $p^{(*)}=p_{0} \lambda^{(*)} \approx 1.625 \mathrm{D}[3,12,13]$. Likewise, for each of the other roots $\gamma_{h, m}$, the criticality condition $\gamma_{h, m}=\gamma^{(*)}=1 / 4$ defines additional, increasingly larger values $\lambda_{h, m}^{(*)}$ of the critical dipole moment. Each of these represents the onset of a new tower of conformal states of the form (7). The sequence of critical values of the dipole moment includes $\lambda_{0,0}^{(*)} \approx 1.279 ; \lambda_{0,1}^{(*)} \approx 7.58 ; \ldots$. However, the experimentally observed bound states $[14,15]$ appear to be limited to the highest root $\gamma_{0,0}$ because of the characteristic order of magnitude of the molecular dipole moments realized in nature.

\section{ROTATIONAL DEGREES OF FREEDOM OF DIPOLE-BOUND ANIONS}

We now turn, through an appropriate length-scale hierarchy, to a derivation of the connection between the approach of Refs. [6,8-10] and the conformal treatment of Ref. [3].

\section{A. Rotationally adiabatic theory} tial

In the rotationally adiabatic theory [9], the pseudopoten- 


$$
\mathcal{V}(r)=-\frac{\hbar^{2}}{2 m_{e}} \frac{\Gamma(\lambda ; F(r))}{r^{2}} G(r)
$$

for the radial electron wave function is an eigenvalue of the reduced Hamiltonian

$$
\hat{\mathcal{H}}=-\frac{\hbar^{2}}{2 m_{e}} \frac{\hat{\mathcal{A}}(\lambda ; F(r))}{r^{2}} G(r),
$$

and the radial function $G(r)$ can be selected by comparison with different expressions used in the literature $[6,8-10]$. In particular, the lowest eigenvalue gives the standard adiabatic potential: $\epsilon_{\text {adiab }}(r) \equiv \mathcal{V}(r)$. In addition, the nontrivial part of the effective Hamiltonian of Eq. (11) arises from the adiabatic approximation for the rotational motion of the molecule, which provides the operator $[6,9,10]$

$$
\hat{\mathcal{A}}(\lambda ; F(r))=-F(r) \mathbf{l}^{2}+\lambda \cos \theta,
$$

where the function $F(r)$ has the form $F(r)=1+\left(r / r_{B}\right)^{2}$, in which the length scale

$$
r_{B}=\sqrt{\frac{\hbar^{2}}{2 m_{e} B}}
$$

is associated with the rotator constant $B=\hbar^{2} / 2 I$ (with $I$ being the moment of inertia). Simple inspection shows that $\hat{\mathcal{A}}(\lambda ; F(r))$ is a generalization of $\hat{A}(\lambda)$, in which the replacement $\mathbf{l}^{2} \rightarrow F(r) \mathbf{l}^{2}$ is made; therefore their angular operator structures are identical. Using again the orbital angular momentum basis $|l, m\rangle$ of the electron, the eigenvalue $\Gamma$ $\equiv \Gamma(\lambda ; F)$ of $\hat{\mathcal{A}}(\lambda ; F)$ can be found from the secular equation

$$
\mathcal{D}_{m}(\Gamma, \lambda ; F(r)) \equiv \operatorname{det}\left[\mathcal{M}_{l l^{\prime}}(\Gamma, \lambda ; m ; F(r))\right]=0,
$$

where $\quad \mathcal{M}(\Gamma, \lambda ; F(r))=-\mathcal{A}(\lambda ; F(r))+\Gamma 1, \quad$ so that $\mathcal{M}_{l l^{\prime}}(\Gamma, \lambda ; m ; F(r))$ is obtained from Eq. (9) by the replacements $l(l+1) \rightarrow l(l+1) F(r)$ and $\gamma \rightarrow \Gamma$ in the diagonal terms. Therefore the eigenvalues arising from Eq. (14) can be labeled just as those derived from the conformal secular determinant: $\Gamma_{h, m}$. In particular, the largest one, $\Gamma_{0,0}$, leads to the standard adiabatic potential $\epsilon_{\text {adiab }}(r)$ $=-\hbar^{2} \Gamma_{0,0}(\lambda ; F(r)) G(r) /\left(2 m_{e} r^{2}\right)$ in Eq. (10).

\section{B. Separation of scales: Renormalization theory}

The current reformulation of the rotationally adiabatic theory permits a direct comparison with the results of the conformal framework, to which it reduces by the use of effective field theory arguments. The reason for this lies in that, in a renormalization treatment, the phenomenological factor $G(r)$ merely amounts to an ultraviolet regulator-only needed for distances $r \lessgtr a$, where $a$ is the size of the molecule. In other words, the details of the position dependence of $G(r)$ are of secondary importance because $G(r) \approx 1$ for $r$ $\gtrsim a$ and the conformal potential effectively dominates the relevant physics. Consequently, the only significant addition to the conformal framework appears to be the inclusion of rotational degrees of freedom via the function $F(r)$. However, a careful analysis of Eq. (14) shows that the conclu- sions from the conformal framework are not substantially altered. The fundamental concept that underlies this surprising result-and which makes our construction successful-is the clear-cut separation of scales. This is the essential assumption that underlies renormalization theory [1], as described in the effective field theory language [2]. Specifically, the two characteristic length scales for the molecular anions are (i) a scale of the order of the molecular size $a$; and (ii) the rotational scale $r_{B}$ of Eq. (13), whose size can be gleaned from $I \sim M a^{2}$, with $M$ being the mass of the molecule. Then, the scale hierarchy

$$
r_{B} \sim \sqrt{\frac{M}{m_{e}}} a \gg a
$$

shows that $L_{\mathrm{UV}} \sim a$, and $L_{\mathrm{IR}} \sim r_{B}$ play the role of "ultraviolet" and "infrared" scales, respectively. Moreover, Eq. (15) provides a justification for the adiabatic approximation used in Refs. $[6,8,9]$; remarkably, this approximation is just a statement about length scales within an effective-field-theory description of molecular physics [16]. Thus the conformal treatment constitutes a satisfactory framework for the physics of dipole-bound molecular anions. This description can be further justified by introducing a systematic reduction procedure. First, the dependence of $\mathcal{V}(r)$ for $r \gg r_{B}$ plays a secondary role for the problem of criticality. This can be rigorously established by an asymptotic analysis of the determinant (14). Most importantly, the existence of a critical value and the ensuing bound states follow from the relevant scales $r \lessgtr r_{B}$ : criticality does not originate in the infrared sector. Second, the critical dipole moment arises from the ultraviolet boundary and can be established by a renormalization framework. Therefore the dominant physics can be extracted by considering the intermediate scales, with $a \leq r$ $\ll r_{B}$. In that range, $F(r) \approx 1$ and $\Gamma(\lambda ; F)$ in Eq. (14) can be replaced by a constant $\gamma(\lambda) \equiv \Gamma(\lambda ; 1)$. Thus, in this "scale window," the adiabatic potential approximately reduces to a long-range conformal potential $\mathcal{V}(r)=-\hbar^{2} \gamma /\left(2 m_{e} r^{2}\right)$. Retracing the previous steps, this reduction establishes the Hamiltonian (1), whose conformal symmetry is reminiscent of the corresponding description in high-energy physics [17]: at sufficiently small distances the problem becomes scale invariant. Finally, when a length scale of the order $a$ is reached, "new physics" emerges and a more detailed treatment is in order-for which a specific form of the factor $G(r)$ would be needed.

\section{GENERALIZED CONFORMAL FRAMEWORK: PREDICTIONS AND NATURE OF THE CORRECTIONS}

The length-scale analysis leads to a noteworthy adjustment to the previous results: the restriction of the conformal tower of bound states to the relevant range of scales. This is due to the fact that the dominant physics is described by a "conformal window" limited by the characteristic scales $L_{\mathrm{UV}}$ and $L_{\mathrm{IR}}$, which act as ultraviolet and infrared cutoffs [5]. The existence of an ultraviolet boundary is directly involved in the renormalization process and drives the fundamental properties of the renormalized conformal framework. By contrast, 
as shown in Ref. [5], the infrared boundary only restricts the range of the dominant physics.

Most importantly, there are a number of predictions arising from this generalized conformal framework, whichwith appropriate refinements-could be tested experimentally and compared against results from alternative approaches. We will illustrate these results by considering the dominant sector of the theory in the subspace $\mathcal{S}_{m=0}(l$ $=0,1)$ of quantum numbers $l=0$ and $l=1$ for the secular determinant (14) with $m=0$, in which $\Gamma_{0,0}=-F+\sqrt{F^{2}+\lambda^{2} / 3}$ [9].

The first prediction arises directly from the existence of a conformal domain, which implies that the number of conformal bound states undergoes a cutoff process leading to a finite value $N_{\text {conf }}$. It turns out that the approximate number

$$
N_{\mathrm{conf}} \sim \frac{\Theta}{\pi} \ln \left(\frac{L_{\mathrm{IR}}}{L_{\mathrm{UV}}}\right)
$$

which is predicted from renormalization, is also in good agreement with known bound-state estimates [18,19]. For typical values of the parameters involved, the logarithmic nature of $N_{\text {conf }}$ yields the generally accepted result that dipole-bound molecular anions sustain only one or two bound states. Therefore, in contrast with the claims of Ref. [6], our approach shows that the presence of a conformal domain is the actual cause for the existence of bound states and of the critical dipole moment.

The second important prediction of the generalized renormalization framework consists of corrections to the critical value $\lambda^{(*)}$. Within the effective-field reduction, as a zerothorder approximation, Eq. (14) [with $F(r) \approx 1]$ provides the required critical dimensionless dipole moment $\lambda_{\text {conf }}^{(*)}$, which is purely conformal in nature. Broadly speaking, when a dipole moment is sufficiently different from the critical value, the predictions of the conformal framework are remarkably accurate. However, very near criticality, $\Theta \sim 0$ and $\kappa \sim 0$; this is due to the fact that the condition of criticality amounts to the emergence of a ground state from the continuum. The corresponding enlarged characteristic size of the ground-state conformal wave function links the relevant scales and corrections are unavoidable in the presence of an infrared cutoff. One possible way of dealing with this is through a perturbative evaluation of $\lambda^{(*)}$ at the level of Eq. (14); nevertheless, because of the extremely long range of the wave function (8), one would have to consider all orders of perturbation theory and carry out infinite resummations. An alternative, more direct estimate can be established from the emergence of the first bound state,

$$
N=N_{\text {conf }}+\delta=1 \text {, }
$$

where $\delta=\delta_{\mathrm{IR}}+\delta_{\mathrm{UV}}$ is the partial contribution of the infrared and ultraviolet sectors to the number of states. The criticality condition (17), combined with Eq. (16), can then be used to evaluate the conformal parameter $\Theta_{\mathrm{gs}}$ of the critical groundstate wave function; the fact that $\Theta_{\mathrm{gs}}$ is small but finite is due to the self-consistent restriction of the theory in the infrared. Thus the fractional correction to the critical dipole value

$$
\epsilon \equiv \frac{\lambda^{(*)}}{\lambda_{\mathrm{conf}}^{(*)}}-1
$$

can be computed from the secular equation (14), by means of Eq. (6), in which $\gamma=1 / 4$ for the purely conformal theory, while $\tilde{\gamma}=\Theta_{\mathrm{gs}}^{2}+1 / 4$ for the theory with an infrared cutoff, so that

$$
\Delta \gamma=\tilde{\gamma}-\frac{1}{4}=\Theta_{\mathrm{gs}}^{2}=4 \pi^{2}(1-\delta)^{2}\left[\ln \left(\frac{r_{B}}{a}\right)^{2}\right]^{-2} .
$$

In particular, in the restriction of the theory to the dominant subspace $\mathcal{S}_{m=0}(l=0,1)$, the quantity $\epsilon$ in Eq. (18) becomes

$$
\epsilon=\sqrt{[1+4(\tilde{\gamma}-\gamma)]\left[1+\frac{4}{9}(\tilde{\gamma}-\gamma)\right]}-1 \approx \frac{20}{9}(\tilde{\gamma}-\gamma),
$$

where the approximate equality arises from the relatively small values of $(\tilde{\gamma}-\gamma)$, which are due to the separation of scales. Consequently, Eqs. (19) and (20) imply that

$$
\epsilon \approx \frac{20}{9} \Theta_{\mathrm{gs}}^{2} \approx \frac{80 \pi^{2}}{9}(1-\delta)^{2}\left[\ln \left(\frac{r_{B}}{a}\right)^{2}\right]^{-2} .
$$

As expected, this correction becomes more prominent for decreasing values of $I$ and increases the critical dipole from its ideal conformal value. In addition, the fractional state contribution $\delta$ in the compensatory factor $(1-\delta)$ can be determined using standard estimates for the number of bound states [19]. With these building blocks, Eq. (21) gives the leading dependence of the critical value $\lambda^{(*)}$ with respect to the infrared scale through $\ln \rho$, with $\rho \equiv I /\left(m_{e} a^{2}\right)=r_{B}^{2} / a^{2}$ being the dimensionless molecular moment of inertia. The logarithmic dependence $\ln \rho$ is the trademark of the underlying renormalization-induced physics and explains the slow convergence of $\lambda^{(*)}$ towards $\lambda_{\text {conf }}^{(*)}$ This analysis ultimately shows that, even when rotational degrees of freedom are included in the description of this problem, renormalization is still responsible for the predicted values of $p^{(*)}$, including:

(i) the existence of a critical value whose order of magnitude is given by the conformal critical point (5); and (21).

(ii) the underlying physics of the logarithmic correction Most importantly, the results (16)-(21) are universal, i.e., model-independent, within the conformal framework.

In addition, we acknowledge the existence of modeldependent corrections to this framework. For molecular dipole anions, these effects can be represented by means of a pseudopotential comprised of electrostatic terms-described by the multipole expansion-combined with many-body contributions of two kinds: a polarization part and an exchange part due to the Pauli exclusion principle $[10,15,20-22]$. The long-distance electrostatic and polarization terms do not substantially affect the rotational infrared corrections to the purely conformal problem because their coupling constants are proportional to $a^{2}$ (with the relevant rotational degrees of freedom being proportional to $r_{B}^{2}$, and $\left.r_{B} \gg a\right)[10,21]$. The short-distance behavior, which contributes to the ultraviolet physics with a scale of the order of 
$L_{\mathrm{UV}} \sim a$, involves electrostatic and exchange many-body effects $[10,21]$. In the case of the exchange effects, the characteristic scale is determined by the overlap of orbitals associated with tightly bound electrons, and the corresponding repulsive core is highly dependent on the nature of the molecular species [23], with $\delta_{\mathrm{UV}}<0$. This negative value partially compensates the positive term $\delta_{\mathrm{IR}}$ and favors the agreement with the observed critical dipole moment in complex molecular species. Consequently, the scale analysis confirms the remarkable fact that the dipole-bound anionic state exists primarily due to the conformal interaction [24]. One of the simplest characterizations of these model-dependent corrections is afforded by the dominant limiting infrared behavior of the rotationally adiabatic theory of Ref. [9], which yields $\delta \approx \delta_{\mathrm{IR}} \approx \sqrt{6} \lambda_{\text {conf }}^{(*)}(1+\epsilon) / 3 \pi$. With these assignments, introducing the parameters $c=\left[\left(\sqrt{6} \lambda_{\text {conf }}^{(*)} / 3 \pi\right)^{-1}-1\right]^{-1} \approx 0.498, A$ $=80 \pi^{2} L^{-2} /\left[9(c+1)^{2}\right]$, and $L=\ln \rho$, the fractional correction to the dipole moment becomes $\epsilon \approx\{[1+1 /(2 c A)]$ $\left.-\sqrt{[1+1 /(2 c A)]^{2}-1}\right\} / c$; for example, for various values of the dimensionless molecular moment of inertia: $\rho=2 \times 10^{8}$, $\rho=2 \times 10^{6}$, and $\rho=4 \times 10^{4}$, the corresponding fractional corrections are, respectively, $\epsilon \approx 0.11, \epsilon \approx 0.16$, and $\epsilon \approx 0.26$ [25].

Finally, let us consider another universal prediction for an experimental realization with at least two conformal bound states [26]. For such a system, Eq. (7) yields the ratio $E_{1} / E_{0}=\exp (-2 \pi / \Theta)$ from which the relative value of the dipole moment, compared to the critical dipole, is

$$
\frac{\lambda}{\lambda^{(*)}}-1 \approx \frac{20}{9} \Theta^{2}=\frac{80 \pi^{2}}{9}\left[\ln \left(\frac{E_{1}}{E_{0}}\right)\right]^{-2},
$$

which can be derived with the restriction to $\mathcal{S}_{m=0}(l=0,1)$, and supplemented by critical-diople corrections just as in Eq. (21). This "inversion" makes a simple prediction solely based on conformal quantum mechanics and which can be explicitly compared against the improved critical value (21), using the known dipole moment $\lambda$ for the given polar molecule. In essence, this is a test of the residual scale invariance of the geometric scaling (7) of the conformal tower of states.

\section{CONCLUSIONS}

In conclusion, the central concept put forward in this paper is the anomalous emergence of bound states via renor- malization for a system with a conformally invariant domain whose ultraviolet boundary dictates binding. The ensuing quantum symmetry breaking within this framework captures the essence of the observed critical dipole moment for the formation of dipole-bound anions.

Moreover, the tools developed in this paper, as exemplified by Eqs. (16)-(22), show that this conformal framework:

(1) permits the extraction of universal properties for physical problems with a conformally invariant domain; and

(2) provides a description of dipole-bound anions in which model-dependent and model-independent contributions can be conveniently organized.

In principle, this generalized conformal framework could be used as the starting point of a systematic approximation scheme for the description of dipole-bound molecular anions. The estimate (21) is a typical illustration of this: its numerical coefficients could be further refined by an improved matching of the conformal domain with the infrared and ultraviolet sectors, as well as by considering higher orders (with respect to $l$ ). Thus our problem is similar to that encountered in many other areas of physics, in which a zeroth order approximation captures the essential ingredients, which are to be subsequently improved upon by the use of miscellaneous approximation techniques.

Most intriguingly, our approach exhibits many similarities with the recently developed chiral-Lagrangian program for nuclear physics $[27,28]$, in which the underlying chiral symmetry from QCD provides a guiding principle within a power-counting scheme that selects the terms in the Lagrangian for nucleons and pions-with the first terms capturing the dominant, model-independent contributions. Likewise, our conformal framework, based on the $\mathrm{SO}(2,1)$ invariance and the use of effective-field theory concepts, is a discriminating scheme to elucidate the dominant model-independent features of the molecular anions and similar systems with a conformally invariant domain; in this context, it would be interesting to develop the analog of the chiral powercounting scheme.

\section{ACKNOWLEDGMENTS}

This work was supported by CONICET, ANPCyT, the University of San Francisco Faculty Development Fund, and by the National Science Foundation under Grants No. 0308300 and No. 0308435.
[1] S. Weinberg, The Quantum Theory of Fields (Cambridge University Press, Cambridge, 1995), Vol. I, Chap. 12.

[2] J. Polchinski, e-print hep-th/9210046.

[3] H. E. Camblong, L. N. Epele, H. Fanchiotti, and C. A. García Canal, Phys. Rev. Lett. 87, 220402 (2001).

[4] H. E. Camblong, L. N. Epele, H. Fanchiotti, and C. A. García Canal, Phys. Rev. Lett. 85, 1590 (2000).

[5] G. N. J. Añaños, H. E. Camblong, and C. R. Ordóñez, Phys. Rev. D 68, 025006 (2003); H. E. Camblong and C. R. Or- dóñez, ibid. 68, 125013 (2003); e-print hep-th/0305035, [Phys. Lett A (to be published)].

[6] M. Bawin, Phys. Rev. A 70, 022505 (2004).

[7] V. Efimov, Phys. Lett. 33B, 563 (1970); Sov. J. Nucl. Phys. 12, 589 (1971); Comments Nucl. Part. Phys. 19, 271 (1990).

[8] W. R. Garrett, Phys. Rev. A 3, 961 (1971); 22, 1769 (1980).

[9] D. C. Clary, J. Phys. Chem. 92, 3173 (1988); and references therein.

[10] H. Abdoul-Carime and C. Desfrançois, Eur. Phys. J. D 2, 149 
(1998), and references therein.

[11] Higher Transcendental Functions, edited by A. Erdélyi and the staff of the Bateman Manuscript Project (McGraw-Hill, New York, 1953), Vol. II, Chap. VII.

[12] E. Fermi and E. Teller, Phys. Rev. 72, 399 (1947); A. S. Wightman, ibid. 77, 521 (1949).

[13] J.-M. Lévy-Leblond, Phys. Rev. 153, 1 (1967); W. Byers Brown and R. E. Roberts, J. Chem. Phys. 46, 2006 (1967); O. H. Crawford, Proc. Phys. Soc. London 91, 279 (1967).

[14] R. D. Mead, K. R. Lykke, W. C. Lineberger, J. Marks, and J. I. Brauman, J. Chem. Phys. 81, 4883 (1984); K. R. Lykke, D. M. Neumark, T. Andersen, V. J. Trappa, and W. C. Lineberger, ibid. 87, 6842 (1987); A. S. Mullin, K. K. Murray, C. P. Schulz, and W. C. Lineberger, J. Phys. Chem. 97, 10281 (1993).

[15] C. Desfrançois, H. Abdoul-Carime, N. Khelifa, and J. P. Schermann, Phys. Rev. Lett. 73, 2436 (1994), and references therein.

[16] J. Moody, A. Shapere, and F. Wilczek, in Geometric Phases in Physics, edited by A. Shapere and F. Wilczek (Singapore, World Scientific, 1989).

[17] R. Jackiw, Phys. Today 25 (1), 23 (1972); Ann. Phys. (N.Y.) 129, 183 (1980); 201, 83 (1990).

[18] F. Calogero, Variable Phase Approach to Potential Scattering (Academic Press, New York, 1967).

[19] In this paper we are using the upper bound $(2 / \pi) \int d r\left|2 m_{e} \mathcal{V}(r) / \hbar^{2}\right|^{1 / 2}$; see Ref. [18].
[20] W. R. Garrett, J. Chem. Phys. 71, 651 (1979).

[21] C. Desfrançois, H. Abdoul-Carime, and J. P. Schermann, Int. J. Mod. Phys. B 12, 1339 (1996).

[22] Similar considerations have been applied to dipolar molecular clusters, for example, in: D. C. Clary and D. M. Benoit, J. Chem. Phys. 111, 10559 (1999); M. Šindelka, V. Špirko, and P. Jungwirth, ibid. 117, 5113 (2002).

[23] In addition to the species dependence, there exist different functional forms of the short-range exchange pseudopotential, as in Refs. [10,21,22].

[24] P. Skurski, M. Gutowski, and J. Simons, J. Chem. Phys. 110, 274 (1999).

[25] The corresponding numerical values in the first article in Ref. [8] (Table I, p. 967) are 0.11, 0.16, and 0.23, respectively (for $R=1.0284 a_{0}$ and $J=0$ ).

[26] Of course, three bound states would provide a more detailed verification of geometrical scaling. However, as in the Efimov effect [7], this is not a likely scenario due to the logarithmic nature of the number (16); see E. Nielsen, D. V. Fedorov, A. S. Jensen, and E. Garrido, Phys. Rep. 347, 373 (2001).

[27] S. Weinberg, Phys. Lett. B 251, 288 (1990); Nucl. Phys. B 363, 3 (1991); Phys. Lett. B 295, 114 (1992).

[28] C. Ordóñez and U. van Kolck, Phys. Lett. B 291, 459 (1992); C. Ordóñez, L. Ray, and U. van Kolck, Phys. Rev. Lett. 72, 1982 (1994); Phys. Rev. C 53, 2086 (1996). 